\title{
Collectivity of the low-lying dipole strength in relativistic random phase approximation
}

\author{
D. Vretenar ${ }^{1,2}$, N. Paar $^{1}$, P. Ring ${ }^{1}$, and G.A. Lalazissis ${ }^{1,3}$ \\ ${ }^{1}$ Physik-Department der Technischen Universität München, \\ D-85748 Garching, Germany \\ ${ }^{2}$ Physics Department, Faculty of Science, University of Zagreb, \\ 10000 Zagreb, Croatia \\ ${ }^{3}$ Physics Department, Aristotle University of Thessaloniki, \\ Thessaloniki GR-54006, Greece
}

November 2, 2018

\begin{abstract}
The relativistic random phase approximation is applied in the analysis of the evolution of the isovector dipole response in nuclei with a large neutron excess. The self-consistent framework of relativistic mean-field theory, which has been very successfully applied in the description of ground-state properties of nuclei far from the valley of $\beta$-stability, is extended to study the possible onset of low-energy collective isovector dipole modes in nuclei with extreme isospin values.
\end{abstract}

Keywords: Relativistic random phase approximation; Relativistic mean-field model; Giant resonances; Pygmy resonances

PACS: 21.60.Jz, 24.30.Cz, 24.30.Gd 


\section{Introduction}

Exotic nuclei with a large neutron excess exhibit unique structure phenomena: the weak binding of the outermost neutrons, pronounced effects of the coupling between bound states and the particle continuum, regions of nuclei with very diffuse neutron densities, the formation of neutron skin and halo structures. The modification of the effective nuclear potential results in a suppression of shell effects, the disappearance of spherical magic numbers, and the onset of deformation and shape coexistence. Isovector quadrupole deformations are expected at the neutron drip-lines, and possible low-energy collective isovector modes have been predicted.

The multipole response of nuclei with large neutron excess has been the subject of many theoretical studies in recent years. In particular, studies of low-energy collective isovector modes provide important information on the isospin and density dependent parts of the effective interactions used in nuclear structure models. Namely, the isoscalar terms of the effective forces are rather well determined by the ground-state properties (sizes, shapes and binding energies). The isovector channel, on the other hand, is much less explored. At present there are many more theoretical predictions than experimental data, and moreover, almost all available data are on light nuclei. For example, low-lying dipole strength was observed for the halo nuclei ${ }^{6} \mathrm{He}$ [四], ${ }^{11} \mathrm{Li}$ [2], and ${ }^{11} \mathrm{Be}$ [3]. For ${ }^{6} \mathrm{He}$ and ${ }^{11} \mathrm{Li}$ about $10 \%$ of the Thomas-ReicheKuhn (TRK) dipole sum rule was found below an excitation energy of 5 $\mathrm{MeV}$. For ${ }^{6} \mathrm{He}$ experimental evidence was also reported for low-lying multipole strength other than dipole. Low-lying dipole strength, exhausting about $5 \%$ of the TRK sum rule for energies up to $5 \mathrm{MeV}$ above the threshold, was

observed for ${ }^{18} \mathrm{O},{ }^{20} \mathrm{O}$ and ${ }^{22} \mathrm{O}$ [1]. The experimental results indicate that, for 
nuclei with a large neutron excess, the strength distribution consists of two major parts. One part corresponds to the real collective excitation, the other at lower excitation energy is caused by single particle excitations of loosely bound neutrons. These results, however, do not exclude the onset of collectivity in the low-energy region for the multipole response of medium-heavy and heavy nuclei.

Most theoretical studies of the multipole response of exotic nuclei have addressed the following problem: What happens to giant resonances in nuclei with a large neutron excess? Catara et al. have studied the low-lying components in strength distributions of weakly bound neutron-rich nuclei [4], and the effect of large neutron excess on the dipole response in the region of the giant dipole resonance in $\mathrm{O}$ and $\mathrm{Ca}$ isotopes [5]. They have shown that the neutron excess increases the fragmentation of the isovector giant dipole resonance (GDR) and that the radial separation of proton and neutron densities leads to non-vanishing isoscalar transition densities to the GDR states. The fragmentation of the isoscalar and isovector monopole strength in neutron rich Ca isotopes has been studied in Ref. [6], and in Ref. [7] the onset of soft dipole resonances in $\mathrm{Ca}$ isotopes has been analyzed. Quite generally, the theoretical analyses have shown that the spectral distributions of exotic nuclei are much more fragmented than those for well bound systems. This happens because protons and neutrons in exotic nuclei feel very different effective potentials, and consequently display very different level spacings. This effect hinders the coherent accumulation of all strength in one dominant resonance mode [8]. In all calculations additional strength has been found below the normal giant resonance region. The detailed predictions for the strength function, however, strongly depend on the effective forces used in the calcu- 
lations. At this stage, therefore, it is important to compare results of various theoretical analyses of the multipole response of exotic nuclei with a large ratio of neutron to proton numbers.

In the present study we apply the relativistic random phase approximation (RRPA) in the analysis of the isovector dipole response of nuclei with a large neutron excess. The self-consistent framework of relativistic meanfield theory, which has been very successfully applied in the description of ground-state properties of nuclei far from the valley of $\beta$-stability, is extended to study the possible onset of low-energy collective isovector modes in nuclei with extreme isospin values.

The low-energy collective isovector dipole mode, i.e. the pygmy resonance, results from the excess neutrons oscillating out of phase with a core composed of equal number of protons and neutrons. A number of theoretical models have been applied in studies of the dynamics of pygmy dipole resonances. These include: the three-fluid hydrodynamical model (the protons, the neutrons of the same orbitals as protons, and the excess neutrons) [9], the two-fluid (the core fluid and the neutron excess fluid) Steinwedel-Jensen hydrodynamical model [10], density functional theory [7], and the Hartree-Fock plus random phase approximation (RPA) with Skyrme forces [5, 11]. More recently, large scale shell model calculations have been performed in studies of pygmy and dipole states in $\mathrm{O}$ isotopes [12], and dipole and spin-dipole strength distributions in ${ }^{11} \mathrm{Li} 13$.

There is also some experimental evidence of possible pygmy dipole states in ${ }^{208} \mathrm{~Pb}$. Studies of the low energy spectrum by elastic photon scattering [14], photoneutron [15], and electron scattering [16] have detected fragmented E1 strength in the energy region between 9 and $11 \mathrm{MeV}$. The fine structure 
exhausts between 3 and $6 \%$ of the E1 sum rule. In a recent study [17] of the isovector dipole response in ${ }^{208} \mathrm{~Pb}$ in the RRPA framework, we have found low-lying E1 peaks in the energy region between 7 and $11 \mathrm{MeV}$. In particular, a collective state has been identified whose dynamics correspond to that of a dipole pygmy resonance.

In this work we extend the analysis of Ref. [17] and study the evolution of collectivity in the isovector dipole response in the low-energy region below the giant dipole resonance. The analysis includes neutron rich isotopes of $\mathrm{O}, \mathrm{Ca}$, $\mathrm{Ni}, \mathrm{Zr}$, and Sn. In Section 2 we present an outline of the relativistic meanfield model and the relativistic random phase approximation. In Section 3 the model is applied in the analysis of the low-lying isovector dipole strength in neutron rich $\mathrm{O}$ and $\mathrm{Ca}$ isotopes. The onset of collective low-energy dipole states, i.e. pygmy resonances, in medium-heavy nuclei is illustrated in Section 4 for $\mathrm{Ni}$ and $\mathrm{Sn}$ isotopes. The results are summarized in Section 5.

\section{Isovector dipole excitations in the relativis- tic RPA}

Models based on quantum hadrodynamics provide a framework in which the nuclear system is described by interacting nucleons and mesons. In comparison with conventional non relativistic descriptions, relativistic models explicitly include mesonic degrees of freedom and consider the nucleons as Dirac particles. A variety of nuclear phenomena have been described in the relativistic framework: nuclear matter, properties of finite spherical and deformed nuclei, hypernuclei, neutron stars, nucleon-nucleus and electronnucleus scattering, relativistic heavy-ion collisions. In particular, relativistic

models based on the mean-field approximation have been successfully applied 
in the description of properties of spherical and deformed $\beta$-stable nuclei, and more recently in studies of exotic nuclei far from the valley of beta stability.

The relativistic mean field theory is based on simple concepts: nucleons are described as point particles, the theory is fully Lorentz invariant, the nucleons move independently in mean fields which originate from the nucleon-nucleon interaction. Conditions of causality and Lorentz invariance impose that the interaction is mediated by the exchange of point-like effective mesons, which couple to the nucleons at local vertices. The single-nucleon dynamics is described by the Dirac equation

$$
\left\{-i \boldsymbol{\alpha} \cdot \boldsymbol{\nabla}+\beta\left(m+g_{\sigma} \sigma\right)+g_{\omega} \omega^{0}+g_{\rho} \tau_{3} \rho_{3}^{0}+e \frac{\left(1-\tau_{3}\right)}{2} A^{0}\right\} \psi_{i}=\varepsilon_{i} \psi_{i} .
$$

$\sigma, \omega$, and $\rho$ are the meson fields, and $A$ denotes the electromagnetic potential. $g_{\sigma} g_{\omega}$, and $g_{\rho}$ are the corresponding coupling constants for the mesons to the nucleon. The lowest order of the quantum field theory is the mean-field approximation: the meson field operators are replaced by their expectation values. The sources of the meson fields are defined by the nucleon densities and currents. The ground state of a nucleus is described by the stationary self-consistent solution of the coupled system of the Dirac (1) and KleinGordon equations. The source terms in the Klein-Gordon equations for the meson fields are calculated in the no-sea approximation, i.e. the Dirac sea of negative energy states does not contribute to the nucleon densities and currents. The quartic potential

$$
U(\sigma)=\frac{1}{2} m_{\sigma}^{2} \sigma^{2}+\frac{1}{3} g_{2} \sigma^{3}+\frac{1}{4} g_{3} \sigma^{4}
$$

introduces an effective density dependence. The non-linear self-interaction of the $\sigma$ field is essential for a quantitative description of properties of finite nuclei. 
By adjusting just a few model parameters: coupling constants and effective masses, to global properties of simple spherical nuclei, it has been possible to describe many nuclear structure phenomena, not only in nuclei along the valley of $\beta$-stability, but also in exotic nuclei with extreme isospin values and close to the particle drip lines. In addition to the self-consistent mean-field potential, pairing correlations have to be included in order to describe ground-state properties of open-shell nuclei. Exotic nuclei, in particular, necessitate a unified description of mean-field and pairing correlations: the relativistic Hartree-Bogoliubov model [18.

In addition to ground state properties, collective excitations have been described in the time-dependent relativistic mean-field model [19, 20, 21] and the relativistic random phase approximation (RRPA) [22, 23, 24, 25]. The RRPA represents the small amplitude limit of the time-dependent relativistic mean-field theory. Self-consistency will therefore ensure that the same correlations which define the ground-state properties, also determine the behavior of small deviations from the equilibrium. The same effective Lagrangian generates the Dirac-Hartree single-particle spectrum and the residual particlehole interaction. In most applications we have used the NL3 interaction [26] for the effective Lagrangian. Properties calculated with the NL3 indicate that this is probably the best effective interaction so far, both for nuclei at and away from the line of $\beta$-stability. This effective interaction will be also used in the present analysis of isovector dipole response in neutron rich nuclei. The RRPA equations form read [23]

$$
\left(\begin{array}{cc}
A^{J} & B^{J} \\
B^{* J} & A^{* J}
\end{array}\right)\left(\begin{array}{c}
X_{\tilde{p} h}^{\nu, J M} \\
Y_{\tilde{p} h}^{\nu, J M}
\end{array}\right)=\omega_{\nu}\left(\begin{array}{cc}
1 & 0 \\
0 & -1
\end{array}\right)\left(\begin{array}{c}
X_{\tilde{p} h}^{\nu, J M} \\
Y_{\tilde{p} h}^{\nu, J M}
\end{array}\right)
$$

The matrix elements contain the single-particle energies and the two-body 
interaction

$$
\begin{gathered}
A_{j_{\tilde{p}} j_{h}, j_{\tilde{q}} j_{i}}^{J}=\left(\epsilon_{j_{\tilde{p}}}-\epsilon_{j_{h}}\right) \delta_{j_{\tilde{p}} j_{\tilde{q}}} \delta_{j_{h} j_{i}}+V_{j_{\tilde{p}} j_{i} j_{h} j_{\tilde{q}}}^{J} \\
B_{j_{\tilde{p}} j_{h}, j_{\tilde{q}} j_{i}}^{J}=(-1)^{j_{\tilde{q}}-j_{i}+J} V_{j_{\tilde{p}} j_{\tilde{q}} j_{h} j_{i}}^{J} .
\end{gathered}
$$

$\tilde{p}$ denotes both particle and antiparticle states, $h$ denotes states in the Fermi sea. An RRPA calculation, consistent with the mean-field model in the no - sea approximation, necessitates configuration spaces that include both particle-hole pairs and pairs formed from occupied states and negative-energy states [23]. The contributions from configurations built from occupied positiveenergy states and negative-energy states are essential for current conservation and the decoupling of the spurious state. In addition, configurations which include negative-energy states give an important contribution to the collectivity of excited states. In a recent study [25] we have shown that, in order to reproduce results of time-dependent relativistic mean-field calculations for giant resonances, the RRPA configuration space must contain negative-energy Dirac states, and the two-body matrix elements must include contributions from the spatial components of the vector meson fields.

The solutions of the RRPA equations (3) are used to evaluate the electric dipole response

$$
B\left(E J, \omega_{\nu}\right)=\frac{1}{2 J+1}\left|\sum_{j_{\tilde{p}} j_{h}} X_{j_{\tilde{p}} j_{h}}^{\nu, J 0}\left\langle j_{\tilde{p}}\left\|\hat{Q}_{J}\right\| j_{h}\right\rangle+(-)^{j_{\tilde{p}}-j_{h}+J} Y_{j_{\tilde{p}} j_{h}}^{\nu, J 0}\left\langle j_{h}\left\|\hat{Q}_{J}\right\| j_{\tilde{p}}\right\rangle\right|^{2} .
$$

for the isovector dipole operator

$$
\hat{Q}_{1 \mu}^{T=1}=\frac{N}{N+Z} \sum_{p=1}^{Z} r_{p} Y_{1 \mu}-\frac{Z}{N+Z} \sum_{n=1}^{N} r_{n} Y_{1 \mu}
$$

A large configuration space is used in order to eliminate the spurious component from the physical states [23]. 
The effect of the Dirac sea states on the isovector dipole strength distribution is not so pronounced as in the isoscalar case [25]. Without the inclusion of the Dirac sea states the position of an isoscalar giant resonances is lowered in energy by several MeV. In contrast, in Fig. 1 1 we illustrate the effect of Dirac sea states on the isovector dipole strength distribution of ${ }^{208} \mathrm{~Pb}$. The solid and long-dashed curves are the RRPA strengths with and without the inclusion of Dirac sea states, respectively. The dotted, dot-dashed and short-dashed curves correspond to calculations in which only the $\sigma$, the $\omega$ and the $\rho$ meson field are included in the coupling between the Fermi sea and Dirac sea states, respectively. We notice that, although the position of the peak is not sensitive to the inclusion of negative energy states, these configurations affect the total intensity, i.e. the values of the calculated energy weighted moments. In particular, if the negative energy Dirac sea states are not included in the RRPA configuration space, only $72.8 \%$ of the energy weighted sum rule is exhausted, as compared with the full RRPA calculation with both positive and negative energy states. When only the $\sigma$, the $\omega$, or the $\rho$ meson couple the particle-hole states with negative energy Dirac sea states, $89.4 \%, 98.3 \%$ and $79.2 \%$ of the full RRPA energy weighted sum rule is exhausted, respectively. The self-consistent RRPA framework used in the present analysis includes configurations built from occupied positive-energy states and negative-energy states.

\section{Low-lying dipole strength in oxygen and calcium isotopes}

In this section the RRPA is applied in the analysis of the low-lying isovector dipole strength in neutron rich $\mathrm{O}$ and $\mathrm{Ca}$ isotopes. The discrete spectra are 
averaged with the Lorentzian distribution

$$
R(E)=\sum_{i} B\left(E 1,1_{i} \rightarrow 0_{f}\right) \frac{\Gamma^{2}}{4\left(E-E_{i}\right)^{2}-\Gamma^{2}},
$$

with the $\mathrm{B}(\mathrm{E} 1)$ values ( 6) calculated for the isovector dipole operator ( [7), and $\Gamma=0.5 \mathrm{MeV}$ is an arbitrary choice for the width of the Lorentzian. The energy of the resonance is defined as the centroid energy

$$
\bar{E}=\frac{m_{1}}{m_{0}}
$$

with the energy weighted moments for discrete spectra

$$
m_{k}=\sum_{i} B\left(E 1,1_{i} \rightarrow 0_{f}\right) E_{i}^{k}
$$

For $k=1$ this equation defines the energy weighted sum rule (EWSR). In the present analysis the EWSR is evaluated in the interval below $50 \mathrm{MeV}$ excitation energy.

In Fig. 2 we display the isovector dipole strength distributions (8) for ${ }^{16} \mathrm{O}$, ${ }^{22} \mathrm{O},{ }^{24} \mathrm{O}$ and the hypothetical nucleus ${ }^{28} \mathrm{O}$. Although ${ }^{24} \mathrm{O}$ is the last bound oxygen isotope, in many mean-field calculations, including the present with the NL3 effective interaction, the neutron drip line is located at ${ }^{28} \mathrm{O}$. Already for ${ }^{16} \mathrm{O}$ the isovector dipole strength distribution is strongly fragmented with the centroid energy at $\bar{E}=21.8 \mathrm{MeV}$. The thin dashed line tentatively separates the region of giant resonances from the low-energy region below 10 $\mathrm{MeV}$. By increasing the number of neutrons, two main effects are observed: a) an increased fragmentation of the dipole strength, and b) the appearance of low lying strength below $10 \mathrm{MeV}$. The relative contribution of the lowenergy region increases with the neutron excess. This is shown in Fig. 3 where we plot the ratios of the energy weighted $m_{1}$ moments calculated in the low $(\mathrm{E} \leq 10 \mathrm{MeV})$ and high $(\mathrm{E}>10 \mathrm{MeV})$ energy regions, as function of 
the neutron excess $N-N_{c}$, with $N_{c}=Z$. In the extreme case of ${ }^{28} \mathrm{O}$, this ratio is almost 0.15 , i.e. a considerable portion of the strength function is located below $10 \mathrm{MeV}$. A similar result for ${ }^{28} \mathrm{O}$ was also obtained in the non-relativistic Hartree-Fock plus RPA framework with Skyrme effective interactions [0]. Several peaks were calculated in the region between 6 and 10 $\mathrm{MeV}$. In the present analysis for ${ }^{28} \mathrm{O}$ the most collective RRPA peak at 15.2 $\mathrm{MeV}$ exhausts $24 \%$ of the EWSR. In the corresponding HF+RPA calculation of Ref. [5], the most collective isovector state exhausts approximately $15 \%$ of the total EWSR.

In a recent experimental investigation, low-lying dipole strength, exhausting around $5 \%$ of the TRK sum rule for energies up to $5 \mathrm{MeV}$ above threshold, was observed for ${ }^{18} \mathrm{O},{ }^{20} \mathrm{O}$ and ${ }^{22} \mathrm{O}$ [1]. Further experimental study of the drip line nucleus ${ }^{24} \mathrm{O}$ is planned in the near future. In the present RRPA calculation we find $2.5 \%, 7.0 \%$ and $8.6 \%$ of the EWSR in the energy region below $10 \mathrm{MeV}$ for ${ }^{22} \mathrm{O},{ }^{24} \mathrm{O}$ and ${ }^{28} \mathrm{O}$, respectively. In comparison, the large scale shell model calculation of Ref. [12] predicts that the low-lying dipole strength below $15 \mathrm{MeV}$ exhausts $10 \%$ of the classical sum rule in ${ }^{22} \mathrm{O}$, and $8.6 \%$ in ${ }^{24} \mathrm{O}$.

What is the nature of these isovector dipole states? The question whether the soft, i.e. low-lying dipole excitations are collective or single-particle has been addressed, for example, in Ref. [27] for the light neutron halo nuclei ${ }^{11} \mathrm{Li}$ and ${ }^{11} \mathrm{Be}$. It has been shown that the soft modes, which result from the large spatial extension of the bound single-particle states, represent a new type of non-resonant independent single-particle excitations. The narrow width and the large transition strength, which characterize these excitations, are not caused by a coherent superposition of particle-hole $(p h)$ configurations like 
in collective states.

In the present RRPA calculation of the oxygen isotopes, we analyze in more detail the structure of the main peaks in the low-energy region of the isovector dipole strength distribution (11). For a state at energy $\omega_{\nu}$, the contribution of a particular proton or neutron $p h$ configuration is determined by the RRPA amplitude

$$
\xi_{\tilde{p} h}=\left|X_{\tilde{p} h}^{\nu}\right|^{2}-\left|Y_{\tilde{p} h}^{\nu}\right|^{2}
$$

with $\mathrm{X}$ and $\mathrm{Y}$ defined by the RRPA equation (3), and the normalization condition

$$
\sum_{\tilde{p} h} \xi_{\tilde{p} h}=1
$$

For ${ }^{22} \mathrm{O}$ we find only one strong peak below $10 \mathrm{MeV}$ : the state at 9.3 MeV exhausts $2.5 \%$ of the EWSR, and its wave function is very simple: single neutron excitations $\left(93 \% 1 d_{5 / 2} \rightarrow 2 p_{3 / 2}\right)$ and $\left(3 \% 1 d_{5 / 2} \rightarrow 1 f_{7 / 2}\right)$. The neutron $p h$ excitations determine also the main peaks in the low-energy region of ${ }^{24} \mathrm{O}$ and ${ }^{28} \mathrm{O}$. For ${ }^{24} \mathrm{O}$ we find three strong peaks at $6.9 \mathrm{MeV}(3.1 \%$ EWSR), $7.4 \mathrm{MeV}$ (1.6\% EWSR) and $9.3 \mathrm{MeV}$ (2.3\% EWSR). These states correspond to the neutron $p h$ excitations: $\left(93 \% 2 s_{1 / 2} \rightarrow 2 p_{3 / 2}\right),\left(96 \% 2 s_{1 / 2} \rightarrow\right.$ $\left.2 p_{1 / 2}\right)$ and $\left(94 \% 1 d_{5 / 2} \rightarrow 2 p_{3 / 2}\right)$, respectively. The strength function below $10 \mathrm{MeV}$ is more fragmented for ${ }^{28} \mathrm{O}$. The main neutron $p h$ components of the states at $4.2 \mathrm{MeV}, 4.9 \mathrm{MeV}, 7.3 \mathrm{MeV}$ and $8.9 \mathrm{MeV}$, are displayed in Table 1. We conclude that in all neutron rich oxygen isotopes the isovector dipole response in the low-energy region below $10 \mathrm{MeV}$ is characterized by single particle transitions, in contrast to the coherent superposition of many $p h$ configurations, which characterizes the excitations in the region of giant resonances. 
The difference in the structure of isovector dipole states in the region below $10 \mathrm{Mev}$ and in the region of giant resonances is illustrated in Fig. 1 , where we display the transition densities for the peaks at $9.3 \mathrm{MeV}$ and 20.9 $\mathrm{MeV}$ in ${ }^{22} \mathrm{O}$, and for the peaks at $7.3 \mathrm{MeV}$ and $18.1 \mathrm{MeV}$ in ${ }^{28} \mathrm{O}$. The proton and neutron contributions are plotted separately; the dotted lines denote the isovector transition densities, and solid lines are used for the isoscalar transition densities. As it has been also shown in Ref. [5], although the isoscalar $\mathrm{B}(\mathrm{E} 1)$ to all states must vanish identically, the corresponding isoscalar transition densities to individual states need not be identically zero. The transition densities for the states at $20.9 \mathrm{MeV}$ in ${ }^{22} \mathrm{O}$ and at $18.1 \mathrm{MeV}$ in ${ }^{28} \mathrm{O}$ display the radial dependence characteristic for the isovector giant dipole resonance: the proton and neutron densities oscillate with opposite phases; the amplitude of the isovector transition density is much larger than that of the isoscalar component; and at large radii both the isovector and isoscalar transition densities have a similar radial dependence. We notice that the large neutron component in the surface region contributes to the formation of a node for the isoscalar transition density. In Ref. [5] it has been shown that this effect is characteristic for neutron rich nuclei.

The transition densities for states in the low-energy region (the states at $9.3 \mathrm{MeV}$ in ${ }^{22} \mathrm{O}$ and at $7.3 \mathrm{MeV}$ in ${ }^{28} \mathrm{O}$ ) exhibit a rather different radial dependence: the proton and neutron densities in the interior region are not out of phase; there is almost no contribution from the protons in the surface region; the isoscalar transition density dominates over the isovector one in the interior; the neutron transition density displays a long tail in the radial coordinate as compared to the transition densities of the giant resonance states. We notice that a similar behavior of transition densities has been 
predicted for ${ }^{6} \mathrm{He},{ }^{11} \mathrm{Li}$ and ${ }^{12} \mathrm{Be}$ in Ref. [28]. It has been shown that the long tails of the wave functions of the loosely-bound neutrons are responsible for the different radial dependence of the soft low-energy states and the giant resonances.

The isovector dipole strength distributions for ${ }^{40} \mathrm{Ca},{ }^{48} \mathrm{Ca},{ }^{54} \mathrm{Ca}$ and ${ }^{60} \mathrm{Ca}$ nuclei are plotted in Fig. 5. As the neutron excess increases, the spectra become more fragmented, and starting with ${ }^{54} \mathrm{Ca}$, the onset of low-lying dipole strength is observed below $10 \mathrm{MeV}$. This result is in agreement with the non-relativistic HF+RPA calculations of Ref. [5]. Notice that no dipole strength is found below $10 \mathrm{MeV}$ for ${ }^{48} \mathrm{Ca}$. In the RRPA strength function of the extremely neutron rich nucleus ${ }^{60} \mathrm{Ca}$, the low-energy region is strongly fragmented, with many peaks of similar intensity. Together, they exhaust $10 \%$ of the EWSR, compared to the $40 \%$ of the EWSR exhausted by the main IV GDR peak at $15.2 \mathrm{MeV}$. The neutron $p h$ configurations with the largest amplitudes in the RRPA wave functions of several low-lying dipole states in ${ }^{60} \mathrm{Ca}$ are listed in Table 2. We notice that one, or at most two neutron ph configurations determine the structure of the low-energy peaks. There is practically no contribution from proton $p h$ excitations. This structure is very different from that of the GDR peak, which is characterized by a coherent superposition of many ph configurations. The largest single neutron ph configuration contributes less than $20 \%$ of the total intensity, and the ratio of the neutron to proton contribution $61.8 \% / 36.7 \%=1.7$ is close to the value $\mathrm{N} / \mathrm{Z}$, as expected for a IV GDR state.

The RRPA transition densities for the state at 7.3 MeV (illustrative for the low-energy region) and for the GDR state at at $15.2 \mathrm{MeV}$, are compared in Fig. 6. The transition densities display the radial dependence and the 
differences that we have already discussed above for the oxygen isotopes. In particular, we notice the long tail of the neutron transition density for the state at $7.3 \mathrm{MeV}$.

Our result for ${ }^{48} \mathrm{Ca}$, i.e. no dipole strength below $10 \mathrm{MeV}$, is at variance with the onset of soft dipole resonances in $\mathrm{Ca}$ isotopes, calculated in the framework of density functional theory [7]. For ${ }^{42,44,46,48} \mathrm{Ca}$, in addition to several narrow peaks which are derived from single particle dipole transitions, a broad resonance was found in the energy range between $5 \mathrm{MeV}$ and $10 \mathrm{MeV}$. It was interpreted as evidence of a collective excitation: surface neutron density oscillating out of phase with a stable ${ }^{40} \mathrm{Ca}$ core. There is also recent experimental evidence on low-energy dipole strength in ${ }^{48} \mathrm{Ca}$. While in experiments with heavy ion reactions [29] no evidence for low-lying strength was found in the comparison of ${ }^{40} \mathrm{Ca}$ and ${ }^{48} \mathrm{Ca}$ spectra, recent results [30] of

high resolution photon scattering experiments indicate the onset of low-lying dipole strength in ${ }^{48} \mathrm{Ca}$. It was found that the sum of the $\mathrm{B}(\mathrm{E} 1)$ strength in the energy region between $5 \mathrm{MeV}$ and $10 \mathrm{MeV}$ is about 10 times larger than in ${ }^{40} \mathrm{Ca}$.

\section{Onset of collective low-energy dipole reso- nances}

Our RRPA analysis of the evolution of the isovector dipole response proceeds in this section towards medium heavy nuclei. The strength distributions (8) for ${ }^{48} \mathrm{Ni},{ }^{56} \mathrm{Ni},{ }^{68} \mathrm{Ni}$ and ${ }^{78} \mathrm{Ni}$ are shown in Fig. 7. Already for ${ }^{48} \mathrm{Ni}$ a peak is found in the low-energy region below $10 \mathrm{MeV}$. This state is characterized by a single proton $p h$ excitation $\left(96 \% 1 f_{7 / 2} \rightarrow 2 d_{5 / 2}\right)$, and it is not found in the spectra of heavier $\mathrm{Ni}$ isotopes. The low-energy dipole states built on 
valence neutron $p h$ configurations appear only in ${ }^{62} \mathrm{Ni}$ and heavier isotopes. The relative contribution of the low-energy region $\mathrm{E} \leq 10 \mathrm{MeV}$ to the dipole strength distribution increases with the neutron excess. The ratio of energy weighted moments $m_{1, \text { low }} / m_{1, \text { high }}$ increases from 0.01 in ${ }^{62} \mathrm{Ni}$ to 0.06 in ${ }^{78} \mathrm{Ni}$ (see Fig. 3). Similar to light nuclei, the low-energy spectra are dominated by single particle transitions. There is an important difference, however. For the heavier $\mathrm{Ni}$ isotopes we find one dipole state in the low-energy region, which displays a more complex structure of the RRPA amplitude, i.e. a coherent superposition of more than just a few neutron $p h$ configurations. In the case of ${ }^{68} \mathrm{Ni}$, this is the state at $9 \mathrm{MeV}(4.3 \%$ EWSR $)$. The distribution of neutron ph configurations for this state is included in Table 3. A dipole state with a similar structure in ${ }^{78} \mathrm{Ni}$ is found at $8.9 \mathrm{MeV}$ (4.0\% EWSR). The transition densities for this state are compared with those of the GDR state at 16.4 $\mathrm{MeV}$ in Fig. 8. We notice that the state at $8.9 \mathrm{MeV}$ is characterized by a strong isoscalar transition density and a long tail of the neutron transition density which extends almost to $10 \mathrm{fm}$.

Suzuki, Ikeda and Sato (SIS) analyzed the onset of pygmy dipole resonances in neutron rich nuclei in the framework of the two-fluid (the core fluid and the neutron excess fluid) Steinwedel-Jensen hydrodynamical model [10]. SIS derived the following relation between the energy of the pygmy dipole resonance and the excitation energy of the GDR

$$
E_{P R}=\sqrt{\frac{Z\left(N-N_{c}\right)}{N\left(Z+N_{c}\right)}} E_{G D R},
$$

where $N_{c}$ denotes the number of neutrons that form the core together with $\mathrm{Z}$ protons, and $\mathrm{N}$ is the total number of neutrons.

In Fig. 9 (upper panel) we compare the RRPA results for the centroid energies of the GDR states in the $\mathrm{Ni}$ isotopes with the empirical relation 
$E=78 A^{-1 / 3}$ [31]. The centroid energies in the low-energy region below $10 \mathrm{MeV}$ are compared with the hydrodynamical prediction for the excitation energies of pygmy resonances (SIS) in the lower panel (eq. (13) with $N_{c}=Z$ ). We notice that the RRPA centroid energies are in fair agreement with the empirical mass dependence of the GDR. The SIS model predicts the position of the pygmy dipole resonance to increase with neutron excess, in contrast to the RRPA results which display the opposite behavior. This discrepancy was also noted and discussed in Ref. [7], where the predictions of the SIS model were compared with results obtained in the framework of microscopic density functional theory.

For the present study a very interesting sequence of medium heavy neutron rich nuclei is the chain of $\mathrm{Sn}$ isotopes with $50 \leq N \leq 82$. In Fig. 10 we display the isovector dipole strength distributions (8) for ${ }^{100} \mathrm{Sn},{ }^{114} \mathrm{Sn}$, ${ }^{120} \mathrm{Sn}$ and ${ }^{132} \mathrm{Sn}$. With the increase of the number of neutrons, the onset of low-lying strength below $10 \mathrm{MeV}$ is observed. The dipole states in this energy region exhibit a structure similar to that observed in the neutron rich Ni isotopes: among several peaks characterized by single particle transitions, between $7 \mathrm{MeV}$ and $9 \mathrm{MeV}$ a state is found with a more distributed structure of the RRPA amplitude, exhausting approximately $2 \%$ of the EWSR. In ${ }^{132} \mathrm{Sn}$, for example, this state is calculated at $8.6 \mathrm{MeV}$ and it exhausts $1.4 \%$ of the EWSR. The distribution of neutron $p h$ configurations for this soft mode is displayed in Table 3. Nine neutron $p h$ configurations contribute with more than $0.1 \%$ to the total RRPA intensity. The total contribution of proton $p h$ excitations is only $10.4 \%$, well below the ratio $\mathrm{Z} / \mathrm{N}$ expected for a GDR state. We notice that also in the Hartree-Fock + RPA analysis of the E1 resonances in ${ }^{208} \mathrm{~Pb}$ [11], it was found that for the pygmy states 
the neutron response is a factor 10 larger than the proton response, whereas at energies corresponding to the GDR this ratio is around 1.6, or roughly $\mathrm{N} /$ Z A similar result was also obtained in our recent RRPA calculation of the isovector dipole response in ${ }^{208} \mathrm{~Pb}$ [17]. In addition to the GDR at 12.95 $\mathrm{MeV}$ and several low-lying single $p h$ states, a collective pygmy dipole state was identified at $7.29 \mathrm{MeV}$, with proton $p h$ excitations contributing only $14 \%$ to the total RRPA intensity.

In Fig. 11 we plot the transition densities to the two states at $8.6 \mathrm{MeV}$ and $14.8 \mathrm{MeV}$ in ${ }^{132} \mathrm{Sn}$. In the upper panel the proton, neutron, isoscalar and isovector components are displayed. The radial dependence and the differences between the transition densities are very similar to those calculated for the pygmy dipole and GDR states in ${ }^{78} \mathrm{Ni}$ (see Fig. 8). In the lower panel, for the pygmy state at $8.6 \mathrm{MeV}$ (c) and for the GDR state at 14.8 $\mathrm{MeV}$ (d), the contributions of the excess neutrons $(50<N \leq 82)$ (solid), and of the proton-neutron core $(Z, N \leq 50)$ (dashed) are displayed separately. By comparing with the transition densities shown in the upper panel of Fig. 11, we notice that there is practically no contribution from the core neutrons $(N \leq 50)$. The ph excitations of core neutrons are, of course, at much higher energies. For the GDR state, therefore, the transition densities of the core nucleons and of the excess neutrons have opposite phases (isovector mode). The absolute radial dependence is similar, with the amplitude strongly peaked in the surface region. The two transition densities have the same sign for the pygmy state at $8.6 \mathrm{MeV}$. The core contribution, however, vanishes for large $r$ and only oscillations of the excess neutrons are observed on the surface of ${ }^{132} \mathrm{Sn}$.

The GDR centroid energies of the Sn isotopes are compared with the 
empirical mass dependence $E=78 A^{-1 / 3}$ [31] in the upper panel of Fig. [12. In the lower panel the centroid energies of the isovector dipole strength in the low-energy region below $10 \mathrm{MeV}$ are compared with the energies of the pygmy dipole states calculated in the two-fluid hydrodynamical model (SIS) [10]. As we have already observed in the case of $\mathrm{Ni}$ isotopes, in contrast to the predicted increase of the pygmy excitation energy with neutron excess in the SIS model, the RRPA centroid energies decrease with the number of neutrons.

As a final example, we analyze the isovector dipole response of a nucleus with an extreme neutron to proton number ratio: ${ }^{122} \mathrm{Zr}(\mathrm{Z}=40, \mathrm{~N}=82)$. The strength distribution is shown in the left panel of Fig. 13. In addition to the GDR structure, the pygmy dipole state is identified at $7.7 \mathrm{MeV}$ excitation energy. This state exhausts $3.3 \%$ of the EWSR. 15 neutron $p h$ configurations contribute with more than $0.1 \%$ to the total RRPA intensity of the pygmy dipole state, compared to 27 neutron $p h$ configurations for the GDR state. The relative contribution of the proton $p h$ excitations is $10.5 \%$ for the pygmy state, and $29.0 \%$ for the GDR state. The transition densities to the pygmy dipole state are displayed in the right panel of Fig. 13. Similar to the structure observed for the pygmy states in $\mathrm{Ni}$ and $\mathrm{Sn}$ isotopes, we notice a very pronounced contribution of the neutron excess in the surface region.

The collective dynamics of the pygmy dipole state is also exemplified in the analysis of transition currents. In Fig. 14 we plot the velocity fields for the pygmy state at $7.7 \mathrm{MeV}$ excitation energy. The velocity distributions are derived from the corresponding transition currents, following the procedure described in Ref. [32]. The velocity field of the proton-neutron core $(Z \leq$ $40, N \leq 50$ ) (left panel), is separated from the contribution of the excess 
neutrons $(50<N \leq 82)$ (right panel). A vector of unit length is assigned to the largest velocity. All the other velocity vectors in both panels are normalized accordingly. The velocity fields of the core nucleons and of the excess neutrons are in phase in the interior region. They have opposite phases in the surface region, though the largest velocities correspond to the vibrations of the excess neutrons on the surface. The core velocities are much smaller than those of the excess neutrons on the surface, in accordance with the transition densities shown in Fig. 13.

\section{Summary}

In the present work we have applied the relativistic random phase approximation (RRPA) in the analysis of the evolution of the isovector dipole response in nuclei with a large neutron excess. The relativistic mean-field model, which has been very successfully applied in the description of ground-state properties of exotic nuclei far from stability, is extended to the RRPA in order to study the possible onset of low-energy collective isovector modes in nuclei with extreme isospin values. Among many new and unique structure phenomena exhibited by nuclei with a large neutron excess, the possible occurrence of collective isovector modes in the energy region below the giant resonances has recently attracted considerable interest. In particular, theoretical and experimental studies have been recently reported on the low-energy collective isovector dipole mode, i.e. the pygmy resonance. The isovector pygmy modes could provide important information on the isospin and density dependence of the effective nuclear interaction. For example, the pygmy dipole resonance can be directly related to the neutron excess, and therefore the splitting between the GDR and the pygmy resonance represents 
a measure of the neutron skin. Precise information on neutron skin in heavy nuclei is essential for the quantification of the isovector channel of effective nuclear forces.

In the analysis of the dynamics of isovector dipole modes we have used the RRPA based on effective mean-field Lagrangians with nonlinear meson self-interaction terms. The NL3 parameter set for the effective mean-field Lagrangian, used in the present calculations, reproduces ground state properties not only in nuclei along the valley of $\beta$-stability, but also in exotic nuclei with extreme isospin values and close to the particle drip lines. The study of the evolution of collectivity in the isovector dipole response in the low-energy region, includes neutron rich isotopes of $\mathrm{O}, \mathrm{Ca}, \mathrm{Ni}, \mathrm{Zr}$, and $\mathrm{Sn}$. We have analyzed the isovector dipole strength distributions, the transition densities, the neutron and proton $p h$ excitations which determine the structure of the RRPA transition amplitudes, the transition currents and velocity distributions of excess neutron and core densities.

The dipole response in nuclei with large neutron to proton ratio is characterized by the fragmentation of the strength distribution and its spreading into the low-energy region, and by the mixing of isoscalar and isovector modes. In light nuclei the onset of dipole strength in the low-energy region is due to single particle excitations of the loosely bound neutrons. In heavier nuclei, low-lying dipole states appear which are characterized by a more distributed structure of the RRPA amplitude, exhausting approximately $2 \%$ of the EWSR. Among several peaks characterized by single particle transitions, a single collective isovector dipole state is identified below $10 \mathrm{MeV}$. A coherent superposition of many neutron particle-hole configurations characterizes its RRPA amplitude. An analysis of the corresponding transition densities 
and velocity distributions reveals the dynamics of the dipole pygmy resonance: the vibration of the excess neutrons against the inert core composed of equal numbers of protons and neutrons. 


\section{Acknowledgments}

This work has been supported in part by the Bundesministerium für Bildung und Forschung under contract 06 TM 979, by the Deutsche Forschungsgemeinschaft, and by the Gesellschaft für Schwerionenforschung (GSI) Darmstadt.

\section{References}

[1] T.Aumann et al., Nucl. Phys. A 649 (1996) 297c.

[2] M. Zinser et al., Nucl. Phys. A 619 (1997) 151.

[3] T. Nakamura et al., Phys. Lett. B 331,(1994) 296.

[4] F. Catara, C.H. Dasso and A. Vitturi, Nucl. Phys. A 602 (1996) 181.

[5] F. Catara, E.G. Lanza, M.A. Nagarajan and A. Vitturi, Nucl. Phys. A $624(1997) 449$.

[6] I. Hamamoto, H. Sagawa and X.Z. Zhang, Phys. Rev. C 56 (1997) 3121.

[7] J. Chambers, E. Zaremba, J.P. Adams and B. Castel, Phys. Rev. C 50 (1994) R2671.

[8] P.-G. Reinhard, Nucl. Phys. A 649 (1999) 305c.

[9] Radhe Mohan, M. Danos and L.C. Biedenharn, Phys. Rev. C 3 (1971) 1740.

[10] Y. Suzuki, K. Ikeda, and H. Sato, Prog. Theor. Phys. 83 (1990) 180.

[11] J.P. Adams, B. Castel and H. Sagawa, Phys. Rev. C 53 (1996) 1016. 
[12] H. Sagawa and Toshio Suzuki, Phys. Rev. C 59 (1999) 3116.

[13] Toshio Suzuki, H. Sagawa and P.F. Bortignon, Nucl. Phys. A 662 (2000) 282.

[14] R.D. Starr, P. Axel and L.S. Cardman, Phys. Rev. C 25 (1982) 780.

[15] Z.W. Bell, L.S. Cardman and P. Axel, Phys. Rev. C 25 (1982) 791.

[16] G. Kühner, D. Meuer, S. Müller, A. Richter, E. Spamer, O. Titze and W. Knüpfer, Phys. Lett. B 104 (1981) 189.

[17] D. Vretenar, N. Paar, P. Ring, and G. A. Lalazissis, submitted to Phys. Rev. C (2000).

[18] G.A. Lalazissis, D. Vretenar, and P. Ring, Nucl. Phys. A650 (1999) 133.

[19] D. Vretenar, H. Berghammer, and P. Ring, Nucl. Phys. A581 (1995) 679.

[20] D. Vretenar, G.A. Lalazissis, R. Behnsch, W. Pöschl and P. Ring, Nucl. Phys. A 621 (1997) 853.

[21] D. Vretenar, N. Paar, P. Ring and G. A. Lalazissis, Phys. Rev. E 60 (1999) 308.

[22] M. L'Huillier and N. Van Giai, Phys. Rev. C 39 (1989) 2022.

[23] F. Dawson and R. J. Furnstahl, Phys. Rev. C 42 (1990) 2009.

[24] D. Vretenar, A. Wandelt, and P. Ring, Phys. Lett. B487 (2000) 334.

[25] Z. Y. Ma, N. Van Giai, A. Wandelt, D. Vretenar and P. Ring, nuclth/9910054. 
[26] G.A. Lalazissis, J. Konig and P. Ring, Phys. Rev. C 55 (1997) 540.

[27] H. Sagawa, N. Van Giai, N. Takigawa, M. Ishihara and K. Yazaki, Z. Phys. A 351 (1995) 385.

[28] H. Sagawa and C. A. Bertulani, Prog. Theor. Phys.(sup.) 124 (1996) 143.

[29] S. Ottini-Hustache, et. al., Phys. Rev. C 59 (1999) 3429.

[30] T. Hartmann, J. Enders, P. Mohr, K. Vogt, S. Volz, and A. Zilges, Phys. Rev. Lett. 85 (2000) 274.

[31] F. E. Bertrand, Nucl. Phys. A354 (1981) 129c.

[32] F. E. Serr, T. S. Dumitrescu, T. Suzuki and C. H. Dasso, Nucl. Phys. A 404 (1983) 359. 
Figure 1: Isovector dipole strength distributions in ${ }^{208} \mathrm{~Pb}$ calculated with the NL3 effective interaction. The solid and long-dashed curves are the RRPA strengths with and without the inclusion of Dirac sea states, respectively. The dotted, dot-dashed and short-dashed curves correspond to calculations in which only the $\sigma$, the $\omega$ and the $\rho$ meson field are included in the coupling between the Fermi sea and Dirac sea states, respectively.

Figure 2: RRPA isovector dipole strength distributions in oxygen isotopes. The thin dashed line tentatively separates the region of giant resonances from the low-energy region below $10 \mathrm{MeV}$.

Figure 3: Ratio of the energy weighted moments $\mathrm{m}_{1}$ in the low-energy region $(\mathrm{E} \leq 10 \mathrm{MeV})$ and in the region of giant resonances $(\mathrm{E}>10 \mathrm{MeV})$. For a number of nuclei the ratio $\mathrm{m}_{1, L O W} / \mathrm{m}_{1, H I G H}$ is plotted as function of the number of excess neutrons.

Figure 4: Transition densities for the peaks at $9.3 \mathrm{MeV}$ and $20.9 \mathrm{MeV}$ in ${ }^{22} \mathrm{O}$, and for the peaks at $7.3 \mathrm{MeV}$ and $18.1 \mathrm{MeV}$ in ${ }^{28} \mathrm{O}$. Both isoscalar and isovector transition densities are displayed, as well as the separate proton and neutron contributions. All transition densities are multiplied by $r^{2}$.

Figure 5: RRPA isovector dipole strength distributions in Ca isotopes. The thin dashed line tentatively separates the region of giant resonances from the low-energy region below $10 \mathrm{MeV}$.

Figure 6: Same as in Fig. 4, but for the peaks at 7.3 MeV and 15.2 MeV in ${ }^{60} \mathrm{Ca}$.

Figure 7: Same as in Fig. 5, but for the Ni isotopes.

Figure 8: Same as in Fig. 4, but for the peaks at 8.9 MeV and $16.4 \mathrm{MeV}$ in ${ }^{78} \mathrm{Ni}$.

Figure 9: Centroid energies( 9) of the isovector dipole strength distribution in the region of giant resonances (upper panel), and in the low-energy region below $10 \mathrm{MeV}$ (lower panel), as functions of the mass number of the $\mathrm{Ni}$ isotopes. The IV GDR centroid energies are compared with the empirical expression $E=78 A^{-1 / 3}$. The energies in the lower panel are compared with the hydrodynamical prediction [10] for the excitation energy of the pygmy resonance (SIS). 
Figure 10: RRPA isovector dipole strength distributions in Sn isotopes. The thin dashed line tentatively separates the region of giant resonances from the low-energy region below $10 \mathrm{MeV}$.

Figure 11: Isovector (IV) and isoscalar (IS) dipole transition densities for the states at $8.6 \mathrm{MeV}$ (a) and $14.8 \mathrm{MeV}$ in ${ }^{132} \mathrm{Sn}$. The separate proton and neutron contributions to the transition densities are also shown. In the lower part of the figure the contributions of the excess neutrons $(50<N \leq 82)$ (solid), and of the proton-neutron core $(Z, N \leq 50)$ (dashed) are displayed separately for the state at $8.6 \mathrm{MeV}$ (c), and $14.8 \mathrm{MeV}$ (d). The transition densities are multiplied by $r^{2}$.

Figure 12: Centroid energies( 9) of the isovector dipole strength distribution in the region of giant resonances (upper panel), and in the low-energy region below $10 \mathrm{MeV}$ (lower panel), as functions of the mass number of the Sn isotopes. The IV GDR centroid energies are compared with the empirical expression $E=78 A^{-1 / 3}$. The energies in the lower panel are compared with the hydrodynamical prediction [10 for the excitation energy of the pygmy resonance (SIS).

Figure 13: Isovector dipole strength distribution in ${ }^{122} \mathrm{Zr}$ (left panel), and transition densities for the peak at $7.7 \mathrm{MeV}$ (right panel). Both isoscalar and isovector transition densities are displayed, as well as the separate proton and neutron contributions (panel b). The contributions of the excess neutrons $(50<N \leq 82)$ (solid), and of the proton-neutron core $(Z \leq 40, N \leq 50)$ (dashed) are displayed separately in panel (c).

Figure 14: Velocity distributions for the RRPA state at $7.7 \mathrm{MeV}$ in ${ }^{122} \mathrm{Zr}$. The velocity field of the proton-neutron core $(Z \leq 40, N \leq 50)$ (left panel), is separated from the contribution of the excess neutrons $(50<N \leq 82)$ (right panel). 
Table 1: For the main peaks in the low-energy region of the isovector dipole strength distribution in ${ }^{28} \mathrm{O}$ (left column), which exhaust a certain percentage of the EWSR (middle column), the neutron particle-hole configurations with largest amplitudes are displayed in the right column. The percentage assigned to a particular $p-h$ configuration refers to the normalization of the RRPA amplitudes (12).

\begin{tabular}{|l|l|l|}
\hline E[MeV] & EWSR[\%] & neutron $p-h$ configuration \\
\hline 4.2 & 0.9 & $\left(92 \% 1 d_{3 / 2} \rightarrow 2 p_{3 / 2}\right)$ \\
\hline 4.9 & 1.4 & $\begin{array}{l}\left(91 \% 1 d_{3 / 2} \rightarrow 2 p_{1 / 2}\right) \\
\left(6 \% 1 d_{3 / 2} \rightarrow 2 p_{3 / 2}\right)\end{array}$ \\
\hline 7.3 & 1.9 & $\left(92 \% 2 s_{1 / 2} \rightarrow 2 p_{3 / 2}\right)$ \\
\hline 8.9 & 6.3 & $\begin{array}{l}\left(71 \% 1 d_{3 / 2} \rightarrow 1 f_{5 / 2}\right) \\
\left(16 \% 1 d_{5 / 2} \rightarrow 1 f_{7 / 2}\right) \\
\end{array}$ \\
& & $\left.3 \% 1 d_{5 / 2} \rightarrow 2 p_{3 / 2}\right)$ \\
\hline
\end{tabular}

Table 2: Same as in Table 1, but for the main peaks in the low-energy region of the isovector dipole strength distribution in ${ }^{60} \mathrm{Ca}$.

\begin{tabular}{|l|l|l|}
\hline E[MeV] & EWSR[\%] & neutron $p-h$ configuration \\
\hline 5.7 & 1.2 & $\left(93.1 \% 2 p_{1 / 2} \rightarrow 3 s_{1 / 2}\right)$ \\
& & $\left(3.6 \% 1 f_{5 / 2} \rightarrow 2 d_{5 / 2}\right)$ \\
\hline 6.8 & 2.6 & $\left(82.8 \% 1 f_{5 / 2} \rightarrow 2 d_{3 / 2}\right)$ \\
& & $\left(6.1 \% 1 f_{5 / 2} \rightarrow 2 d_{5 / 2}\right)$ \\
& & $\left(3.1 \% 1 f_{7 / 2} \rightarrow 1 g_{9 / 2}\right)$ \\
& & $\left(2.3 \% 2 p_{3 / 2} \rightarrow 2 d_{5 / 2}\right)$ \\
& & $\left(1.1 \% 2 p_{3 / 2} \rightarrow 3 s_{1 / 2}\right)$ \\
\hline 7.3 & 1.6 & $\left(49.3 \% 2 p_{1 / 2} \rightarrow 2 d_{3 / 2}\right)$ \\
& & $\left(41.3 \% 2 p_{3 / 2} \rightarrow 3 s_{1 / 2}\right)$ \\
& & $\left(3.2 \% 1 f_{5 / 2} \rightarrow 2 d_{3 / 2}\right)$ \\
& & $\left(1.5 \% 2 p_{3 / 2} \rightarrow 2 d_{5 / 2}\right)$ \\
\hline 7.9 & 2.0 & $\left(83.8 \% 2 p_{3 / 2} \rightarrow 2 d_{5 / 2}\right)$ \\
& & $\left(5.7 \% 1 f_{5 / 2} \rightarrow 2 d_{3 / 2}\right)$ \\
& & $\left(3.5 \% 1 f_{7 / 2} \rightarrow 1 g_{9 / 2}\right)$ \\
& & $\left(2.4 \% 1 f_{5 / 2} \rightarrow 1 g_{7 / 2}\right)$ \\
\hline 9.9 & 2.1 & $\left(57.7 \% 1 f_{5 / 2} \rightarrow 1 g_{7 / 2}\right)$ \\
& & $\left(22.0 \% 1 f_{7 / 2} \rightarrow 1 g_{9 / 2}\right)$ \\
& & $\left(3.3 \% 1 f_{7 / 2} \rightarrow 2 d_{5 / 2}\right)$ \\
\hline
\end{tabular}


Table 3: Distribution of neutron particle-hole configurations for the states at $9.0 \mathrm{MeV}(4.3 \% \mathrm{EWSR})$ in ${ }^{68} \mathrm{Ni}$ (left column), and at $8.6 \mathrm{MeV}$ state $(1.4 \%$ EWSR) in ${ }^{132} \mathrm{Sn}$ (right column). The percentage of a $p-h$ configuration refers to the normalization of the RRPA amplitudes (12). Only configurations which contribute more than $0.1 \%$ are displayed.

\begin{tabular}{|l||l|}
\hline${ }^{68} \mathrm{Ni}$ at $9.0 \mathrm{MeV}$ & ${ }^{132} \mathrm{Sn}$ at $8.6 \mathrm{MeV}$ \\
\hline$\left(26.1 \% 1 f_{5 / 2} \rightarrow 2 d_{5 / 2}\right)$ & $\left(28.2 \% 2 d_{3 / 2} \rightarrow 2 f_{5 / 2}\right)$ \\
$\left(22.9 \% 2 p_{3 / 2} \rightarrow 2 d_{5 / 2}\right)$ & $\left(21.9 \% 2 d_{5 / 2} \rightarrow 2 f_{7 / 2}\right)$ \\
$\left(11.3 \% 1 f_{7 / 2} \rightarrow 1 g_{9 / 2}\right)$ & $\left(19.7 \% 2 d_{3 / 2} \rightarrow 3 p_{1 / 2}\right)$ \\
$\left(10.3 \% 2 p_{1 / 2} \rightarrow 2 d_{3 / 2}\right)$ & $\left(10.5 \% 1 h_{11 / 2} \rightarrow 1 i_{13 / 2}\right)$ \\
$\left(10.0 \% 1 f_{5 / 2} \rightarrow 2 d_{3 / 2}\right)$ & $\left(3.5 \% 2 d_{5 / 2} \rightarrow 3 p_{3 / 2}\right)$ \\
$\left(8.2 \% 2 p_{3 / 2} \rightarrow 3 s_{1 / 2}\right)$ & $\left(1.9 \% 1 g_{7 / 2} \rightarrow 2 f_{5 / 2}\right)$ \\
$\left(1.4 \% 2 p_{1 / 2} \rightarrow 3 s_{1 / 2}\right)$ & $\left(1.5 \% 1 g_{7 / 2} \rightarrow 1 h_{9 / 2}\right)$ \\
$\left(1.0 \% 1 f_{5 / 2} \rightarrow 1 g_{7 / 2}\right)$ & $\left(0.6 \% 1 g_{7 / 2} \rightarrow 2 f_{7 / 2}\right)$ \\
$\left(0.3 \% 1 s_{5 / 2} \rightarrow 3 d_{3 / 2}\right)$ & $\left(0.6 \% 2 d_{3 / 2} \rightarrow 3 p_{3 / 2}\right)$ \\
\hline
\end{tabular}




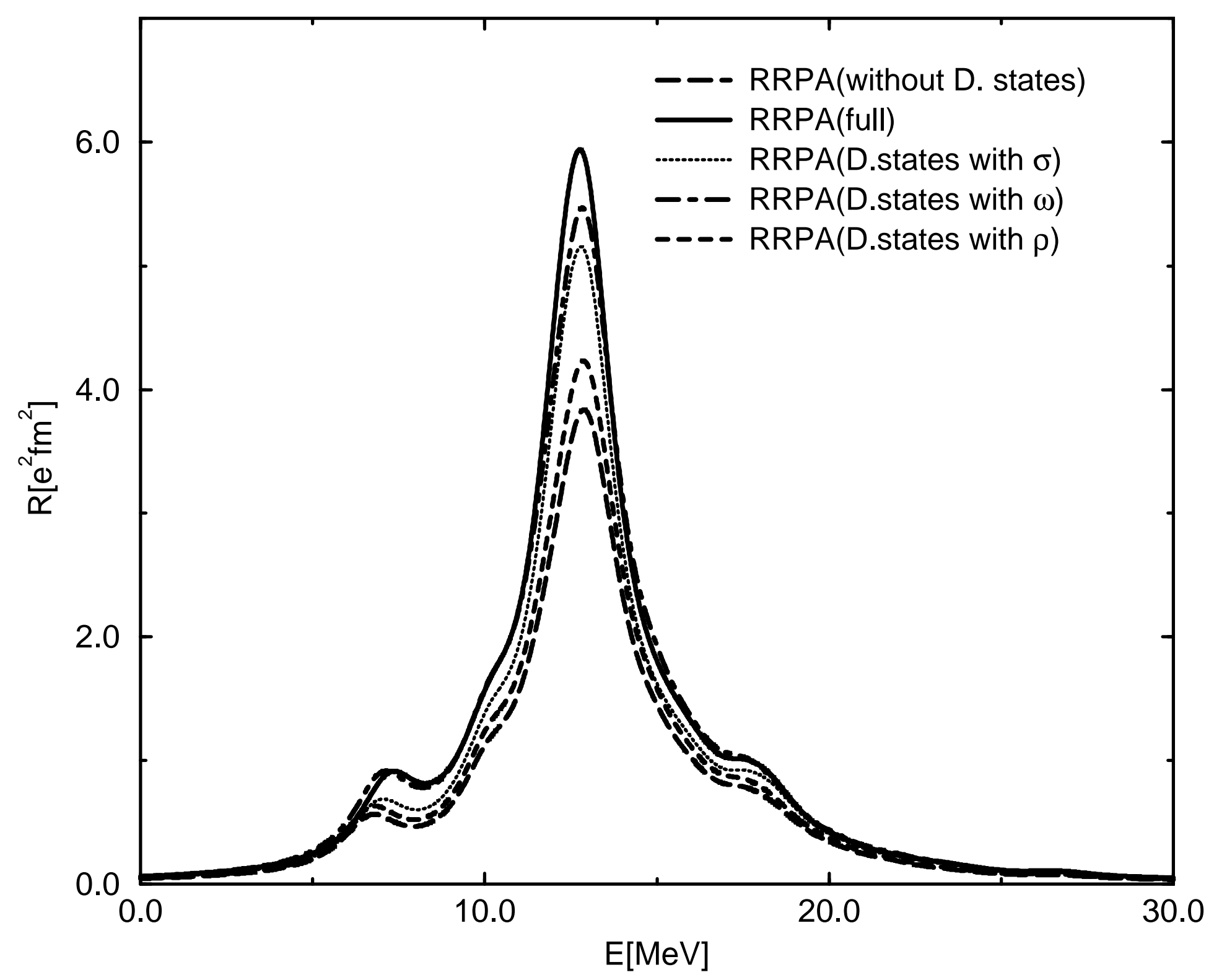




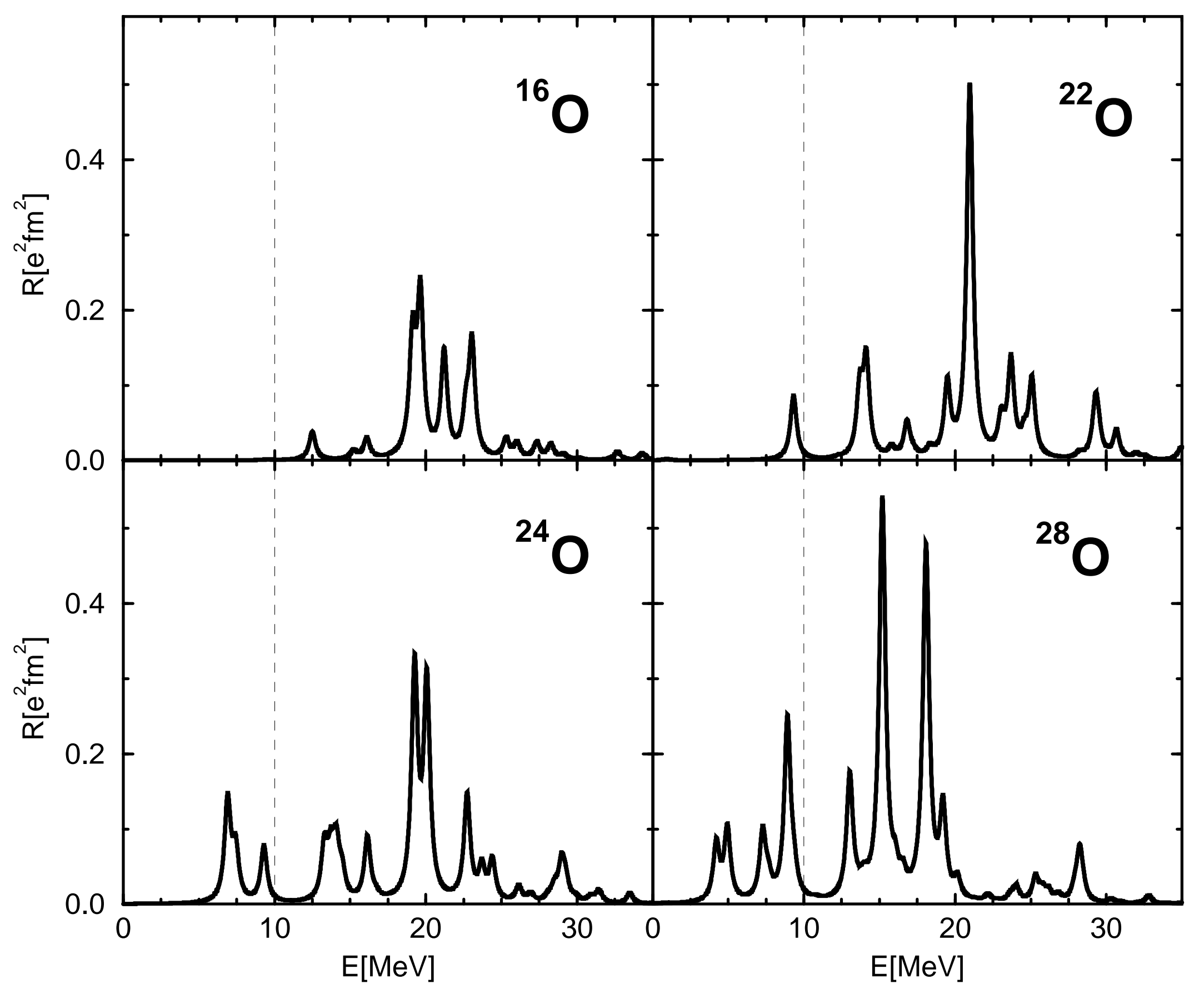




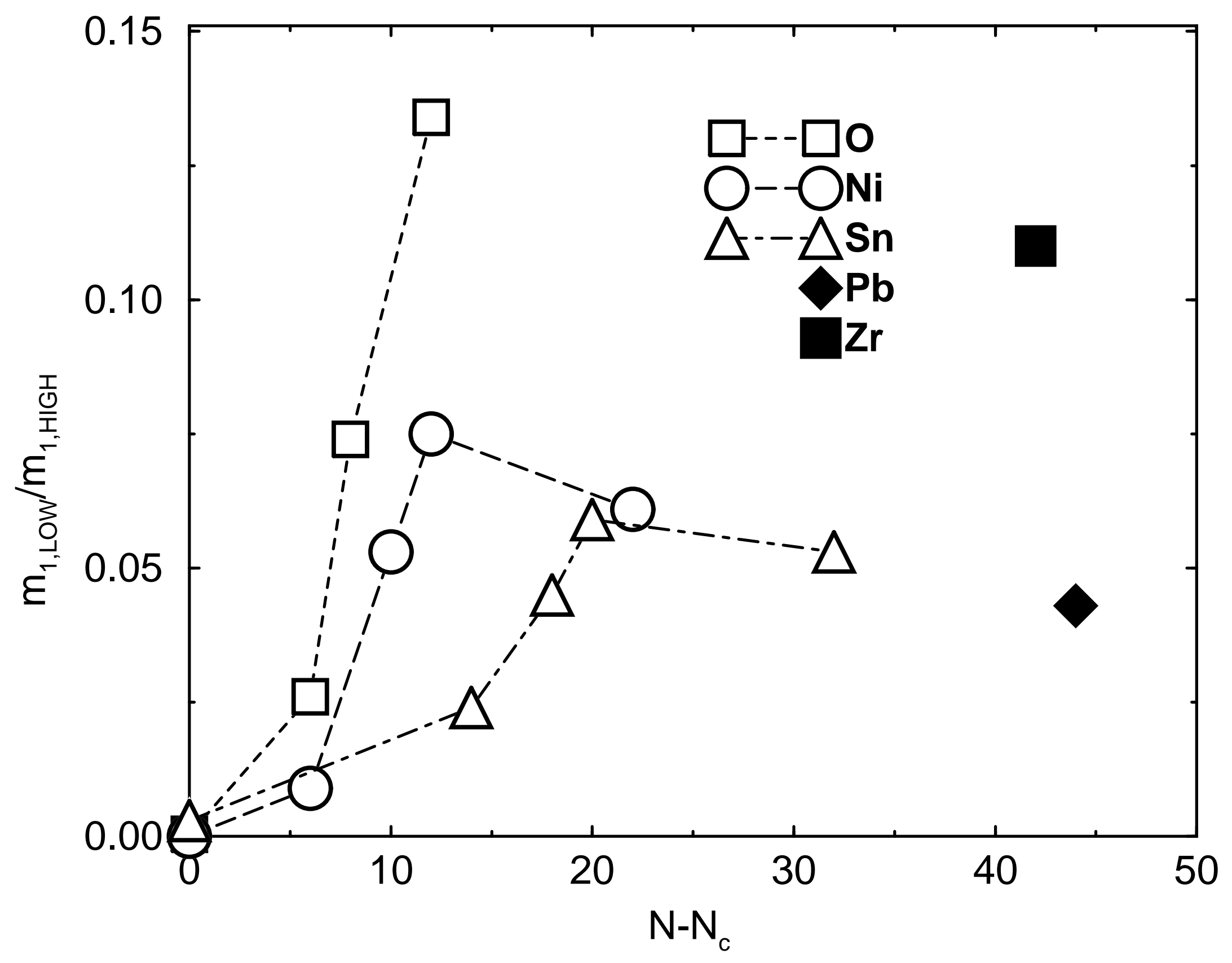









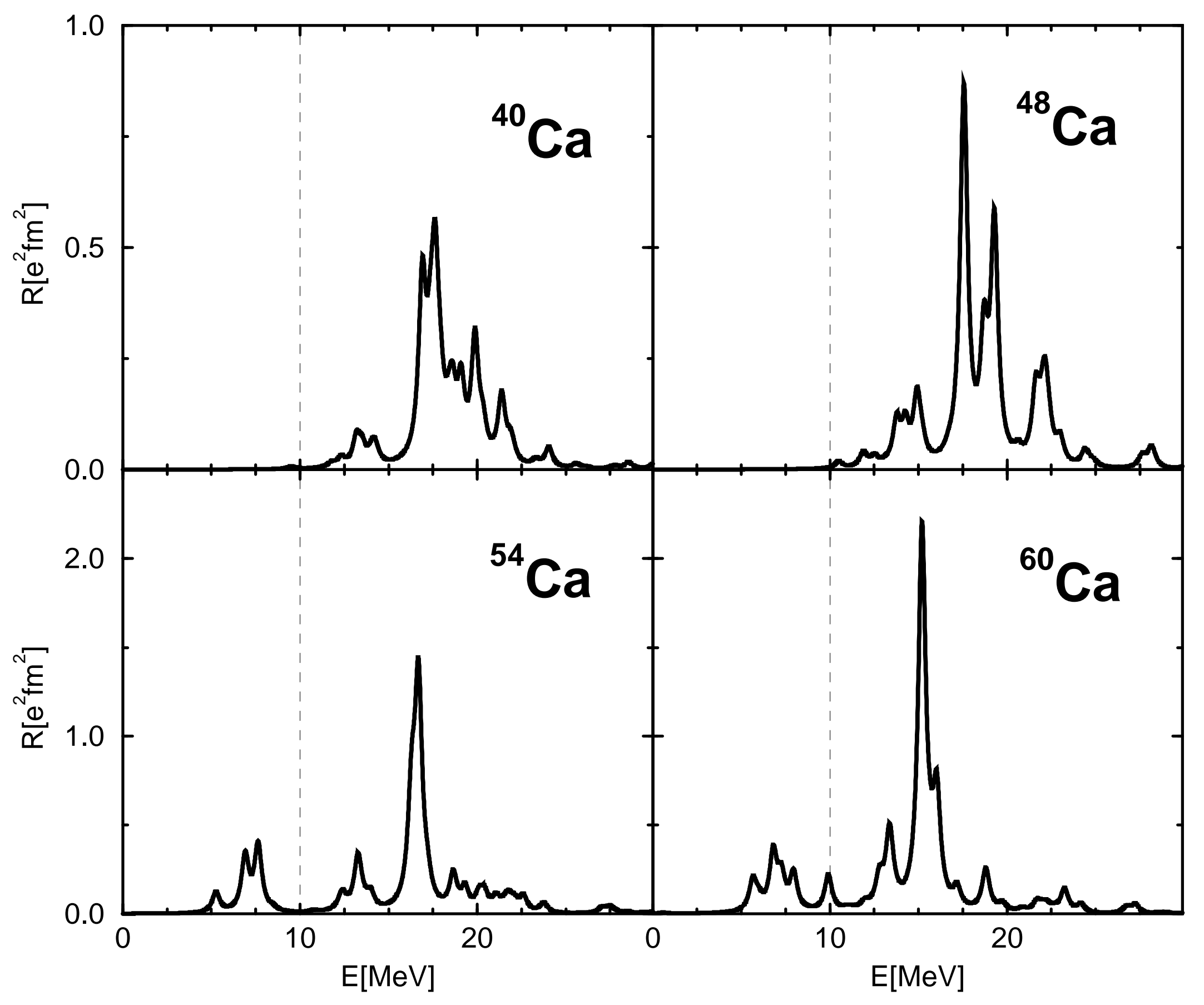




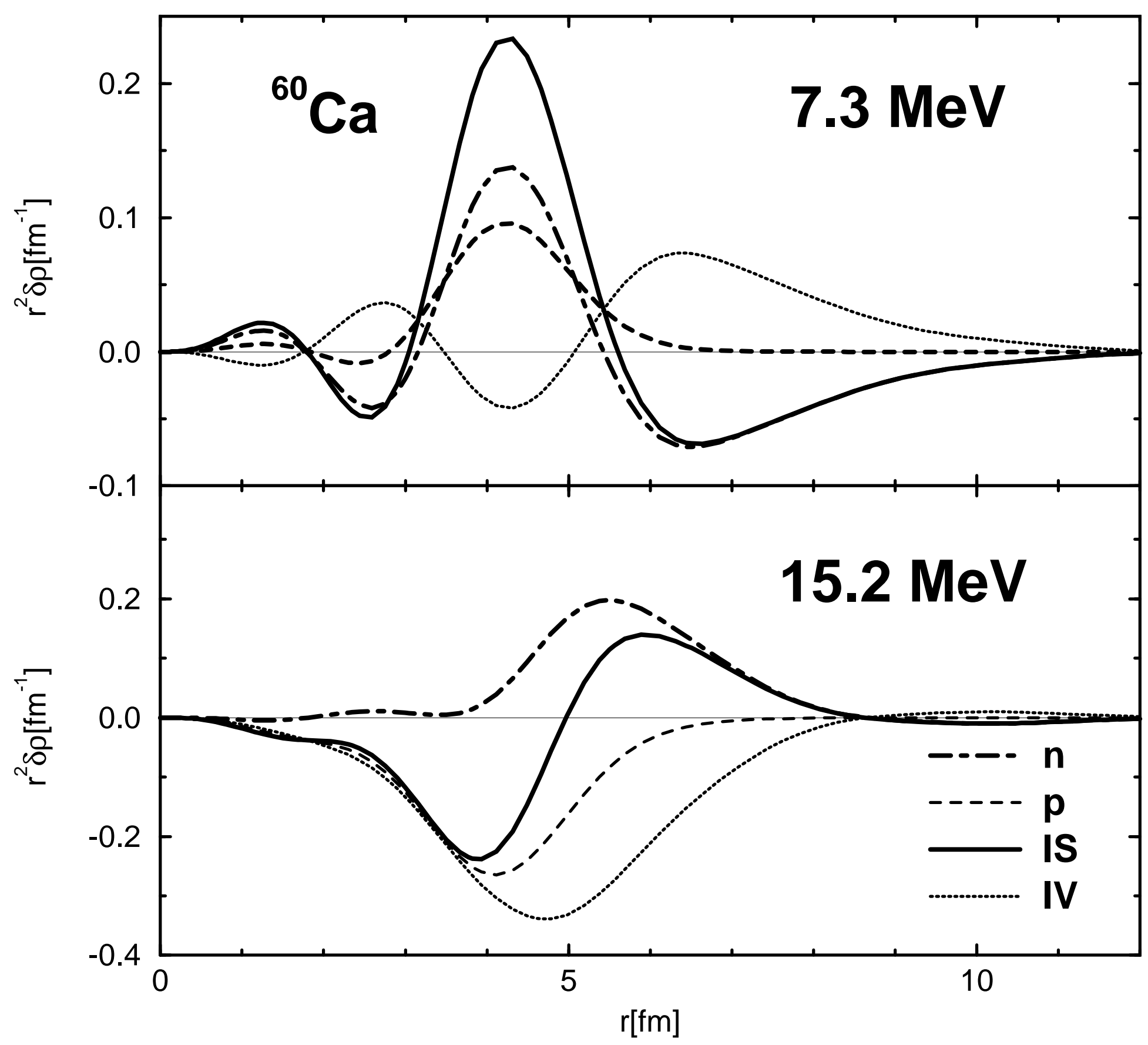




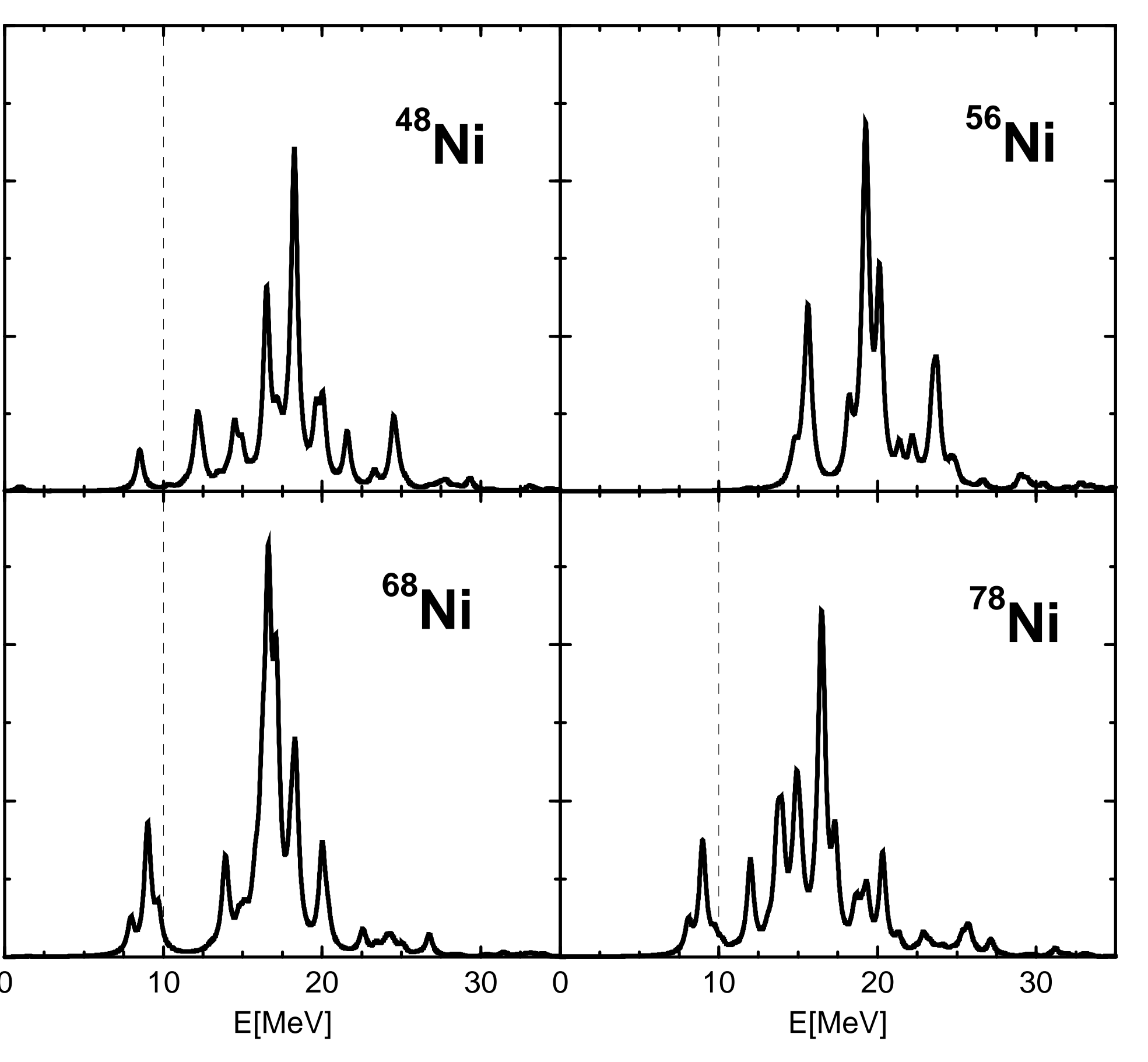




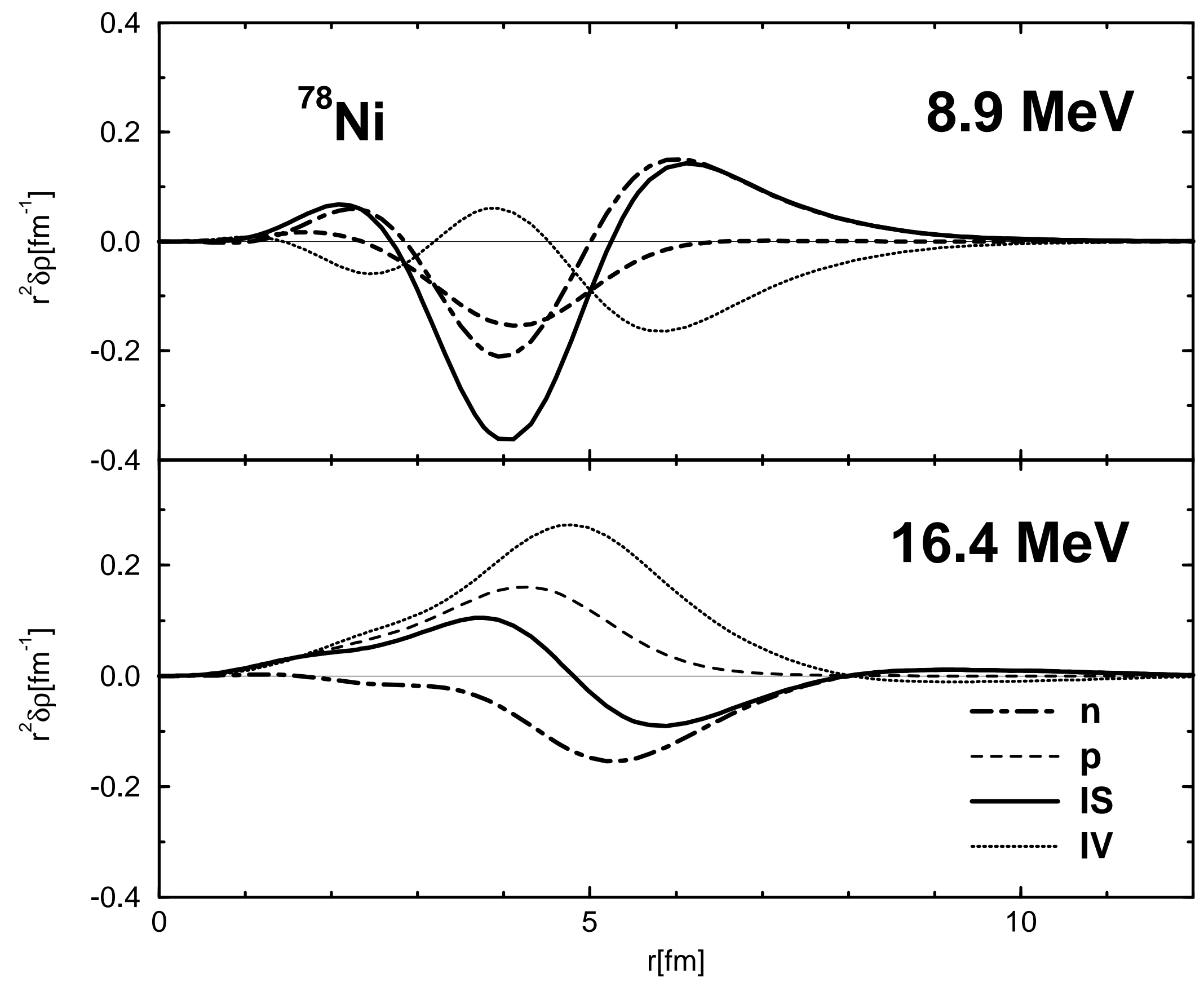









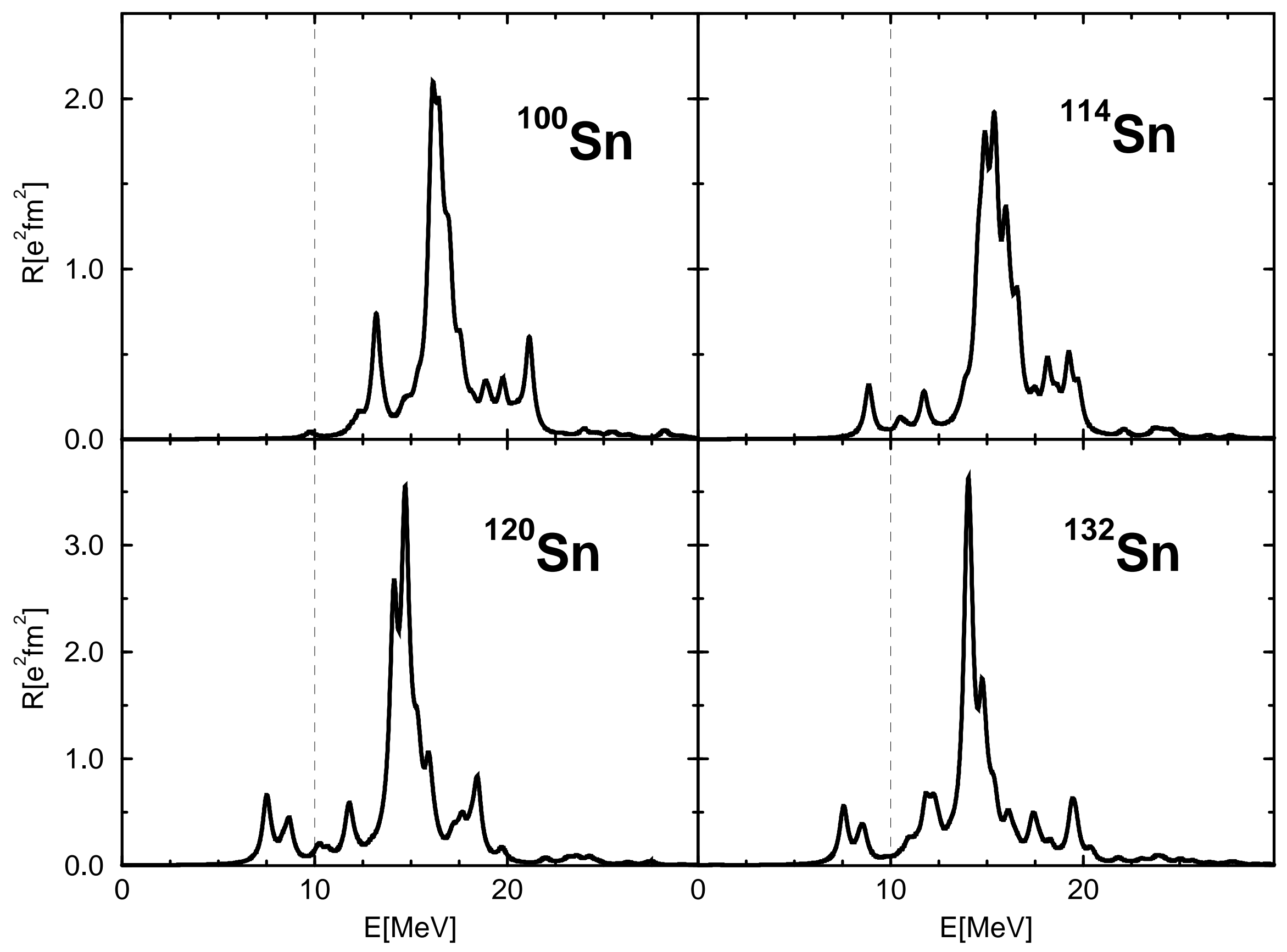




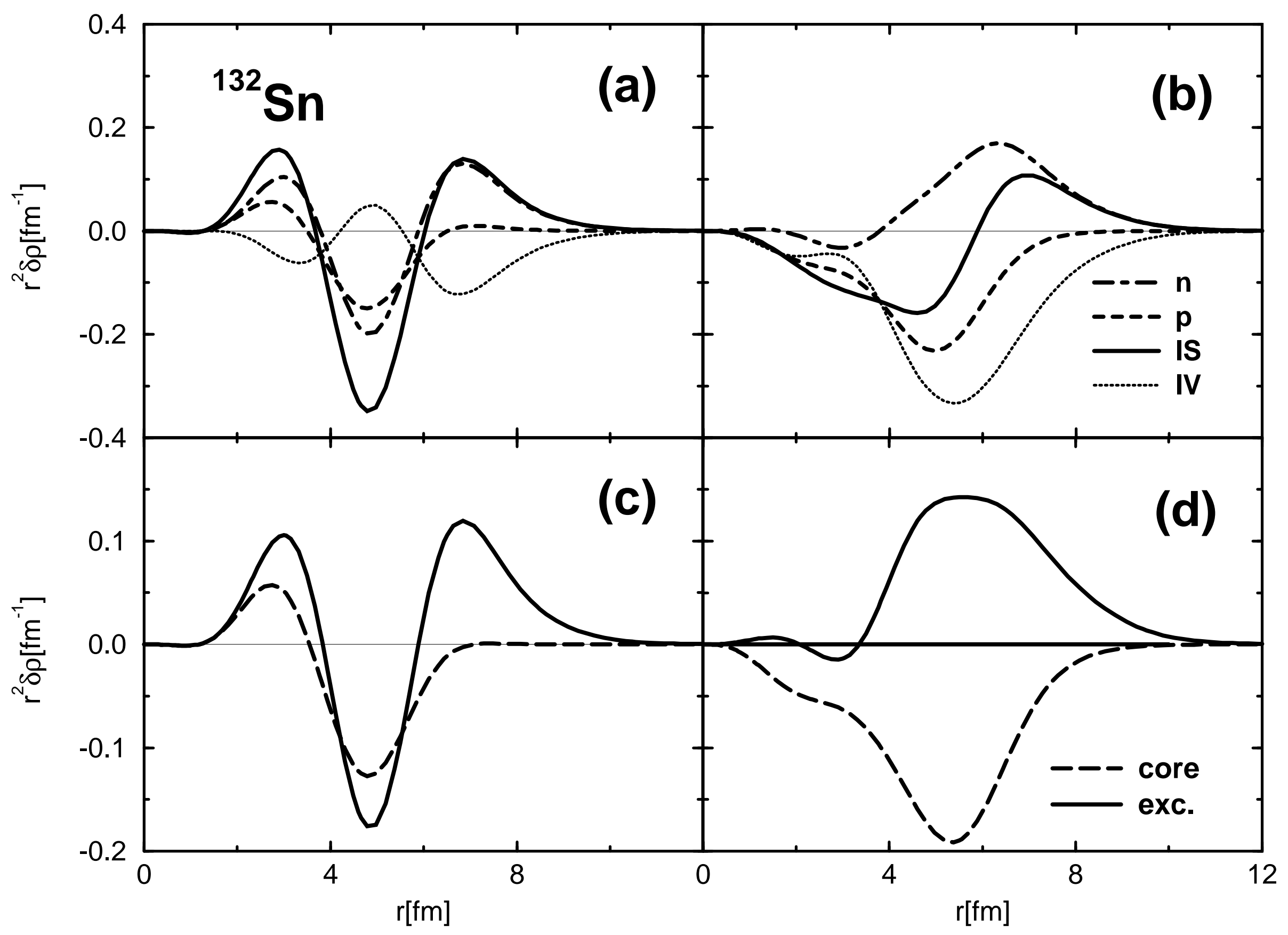




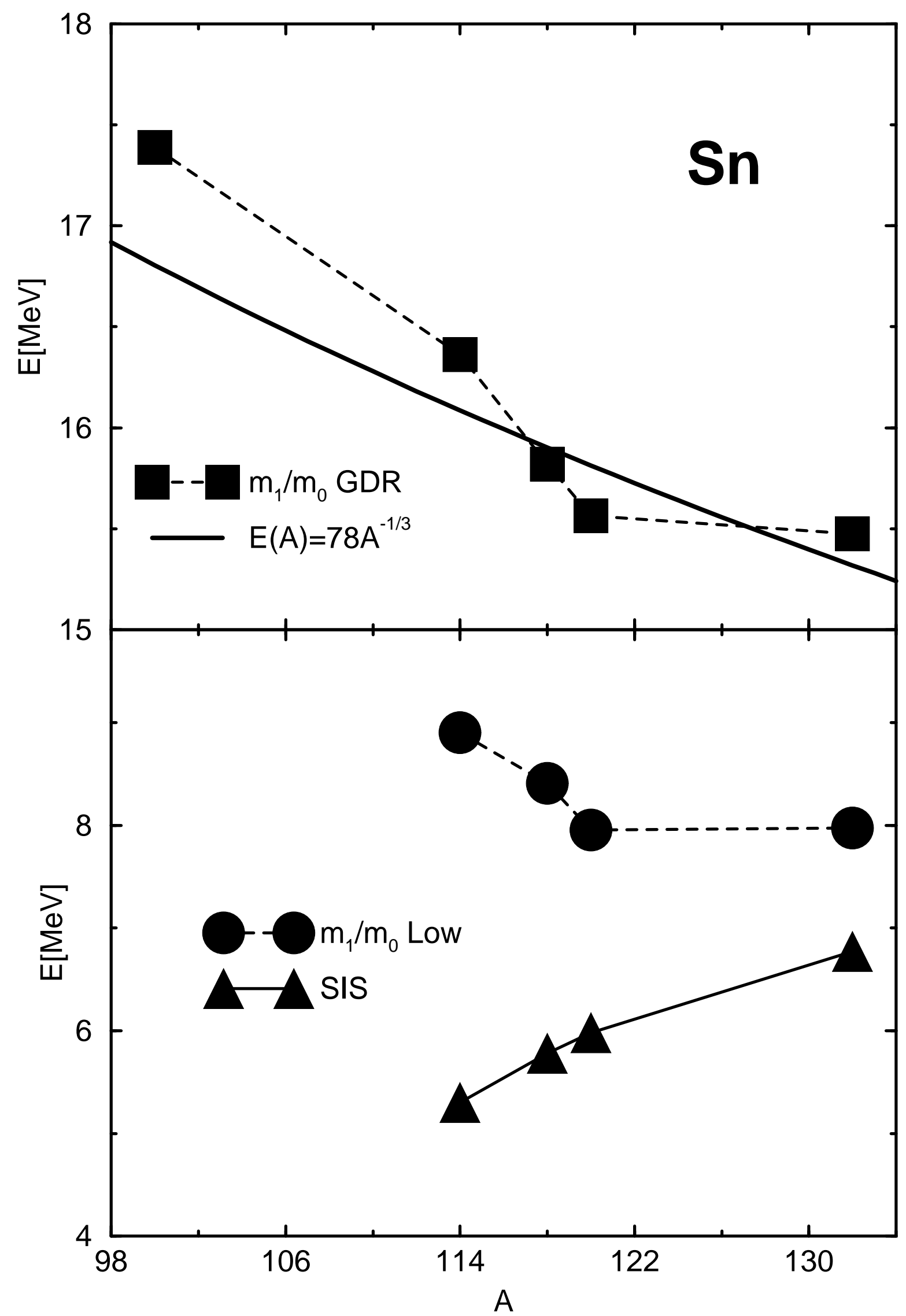








